\title{
36. THE MOTION OF PERIODIC COMET PONS-BROOKS,
}

\section{2-1954}

\author{
P. HERGET and H. J. CARR \\ University of Cincinnati Observatory, Cincinnati, Ohio, U.S.A.
}

\begin{abstract}
The orbit of P/Pons-Brooks has been studied using the observations of 1812, 1883-1884 and 1953-1954. A curious deviation of the residuals has been established during the last two months of observation in April and May 1884, but no discontinuity in the orbit due to an impulsive action on the comet has been found. The observations covering two complete revolutions cannot be fitted into one single orbit.
\end{abstract}

In 1939 the observations of $\mathrm{P} /$ Pons-Brooks were treated in much the same manner as Cowell and Crommelin had used to predict the return of Halley's Comet in 1909. The observations of 1883-1884 were collected only for those observatories which had made 40 or more, so that systematic errors could be examined, but eventually no corrections on this basis seemed justified. These observations were first collected into normal places which were separated by each Full Moon, but they subsequently were replaced by normal places extending over an interval of about ten days each. All the comparison star positions were examined and compared with the Geschichte des Fixsternhimmels in order to provide improved positions whenever possible. The perturbations in rectangular coordinates during the interval of observations were computed by Encke's method. The differential correction process was by Herget's (1939) extension of the Eckert-Brouwer method. The dynamical variables used were $\mathrm{d} T, \mathrm{~d} e$, and $\mathrm{d} a / a$. The equations of condition were solved only for five unknowns in terms of the residuals $(\mathrm{O}-\mathrm{C})$ and $\mathrm{d} a / a$ (since the latter was otherwise nearly indeterminate).

The orbit represented by the best preliminary fit to the observations was then integrated backward to 1812 in terms of the barycentric, rectangular coordinates. The normal places which had been provided by Schulhof and Bossert for the observations of 1812 were then used to determine only the value of $\mathrm{d} a / a$. In other words, on the assumption that the observations of 1812 were of poorer quality, the values of the five unknowns in the equations of condition for 1812 were eliminated in terms of the solution obtained from 1883-1884, and only $\mathrm{d} a / a$ remained in the equations. It was now well determined because an entire period had been observed, and thus the finally adopted orbit for 1883-1884 could be derived.

At this point it was apparent that all the observations in the Southern Hemisphere during the last two months of observation were of increasingly poorer quality. The residuals of the individual observations in the last normal place spread over a circle more than $20^{\prime \prime}$ in diameter and they all lay on one side of the finally adopted path. It seemed futile to attempt at that time to determine the effect of any nongravitational action due to the perihelion passage. Therefore the adopted trajectory was integrated forward to 1954, entirely disregarding what was known of the poor fit at the end of 
the observed arc in 1884 . After the recovery in 1953, the predicted perihelion passage proved to be in error by 4.5 days.

After the Prague meetings of IAU Commission 20 in 1967, we undertook to determine the magnitude of an assumed discontinuity in the gravitational trajectory which must have taken place shortly after perihelion passage in 1884 . It was proposed to determine for Case I an orbit from the observations in 1812 and those before perihelion in 1884, and for Case II a second orbit from those in 1953-1954 and after perihelion in 1884. Then the representation of the observations in 1883-1884 after perihelion as given by Case I, and those before perihelion as given by Case II would be an indication of the magnitude of the discontinuity.

Our efforts, however, produced a different result. Along with the Schubart-Stumpff $N$-body integration program for the trajectory of the comet, we integrated the variational equations in terms of the starting values, $x_{0}, y_{0}, z_{0}, x_{0}^{\prime}, y_{0}^{\prime}$, and $z_{0}^{\prime}$, with the epoch near perihelion at JD 2409200.5. Normal places were abandoned, and each observation was used individually. Both Case I and Case II, separately, showed that the observations in the Southern Hemisphere during the last two months deviated in nearly the same fashion as had been found earlier. Therefore these observations were not used in what follows. Case $\mathrm{A}$ is a differential correction based on all the remaining observations of 1883-1884 and 1953-1954, and Case B is a differential correction based on all the remaining observations of 1883-1884 and the six normal places of 1812. The residuals of all the observations of 1883-1884 show an almost identical pattern in both Cases. The unit vectors perpendicular to the two orbit planes differ in direction by less than one second of arc. The starting values of both Cases and the differences of the position and velocity vectors at the epoch are as shown in Table I. Between the two Cases: $(\mathrm{d} P / P)=0.0001702=4 \mathrm{~d} 37$. With this disparity, we have

TABLE I

Position and velocity components for the epoch JD 2409200.5

\begin{tabular}{llr}
\hline Case B & Case A & \multicolumn{1}{c}{ Diff. } \\
\hline+0.132082942748 & +0.132082068306 & +874442 \\
+0.758828947641 & +0.758829039873 & -92232 \\
+0.094968656120 & +0.094969079510 & -423390 \\
$(40 \mathrm{k}) \bar{v}_{0}$ & $(40 \mathrm{k}) \bar{v}_{0}$ & \\
-0.362633594277 & -0.362633469038 & -125239 \\
+0.166990147726 & +0.166988666760 & +1480966 \\
-1.016524295006 & -1.016523056834 & -1238172 \\
\hline
\end{tabular}

found it impossible to combine the observations of all three apparitions into one orbit. The increasingly wide systematic deviations of the observations during the last two months in 1884 also remain unexplained. All the residuals are shown in Figures 1-3, except those of 1812, which are given in Table II. Their systematic character also shows that our results are far from satisfactory. Undoubtedly there must be nongravitational forces acting, but we feel that the distribution of observations is such that the arbitrary introduction of additional parameters is not justified. 

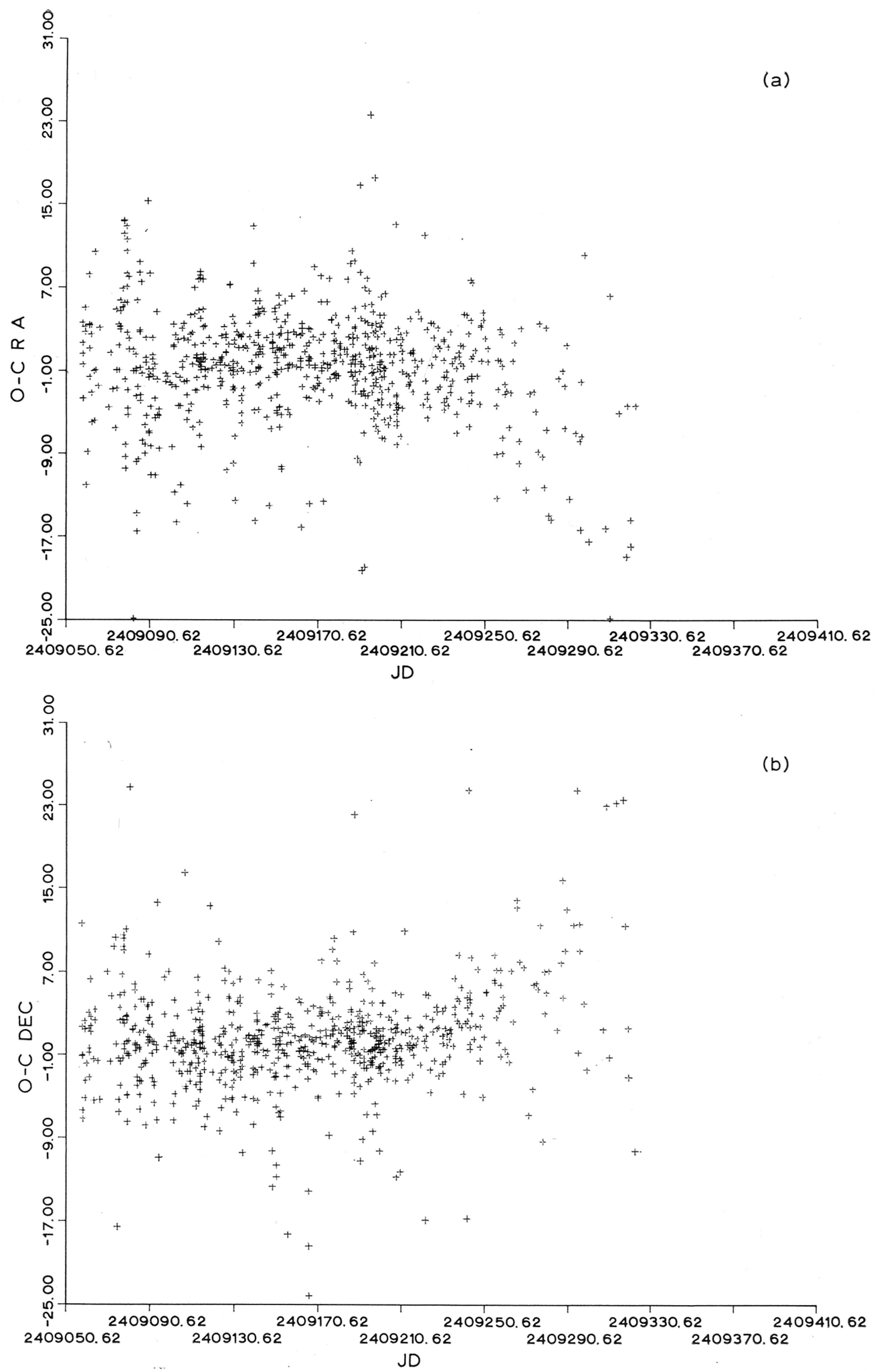

Figs. 1a-b. (O-C) residuals in 1883-1884; Case A. 

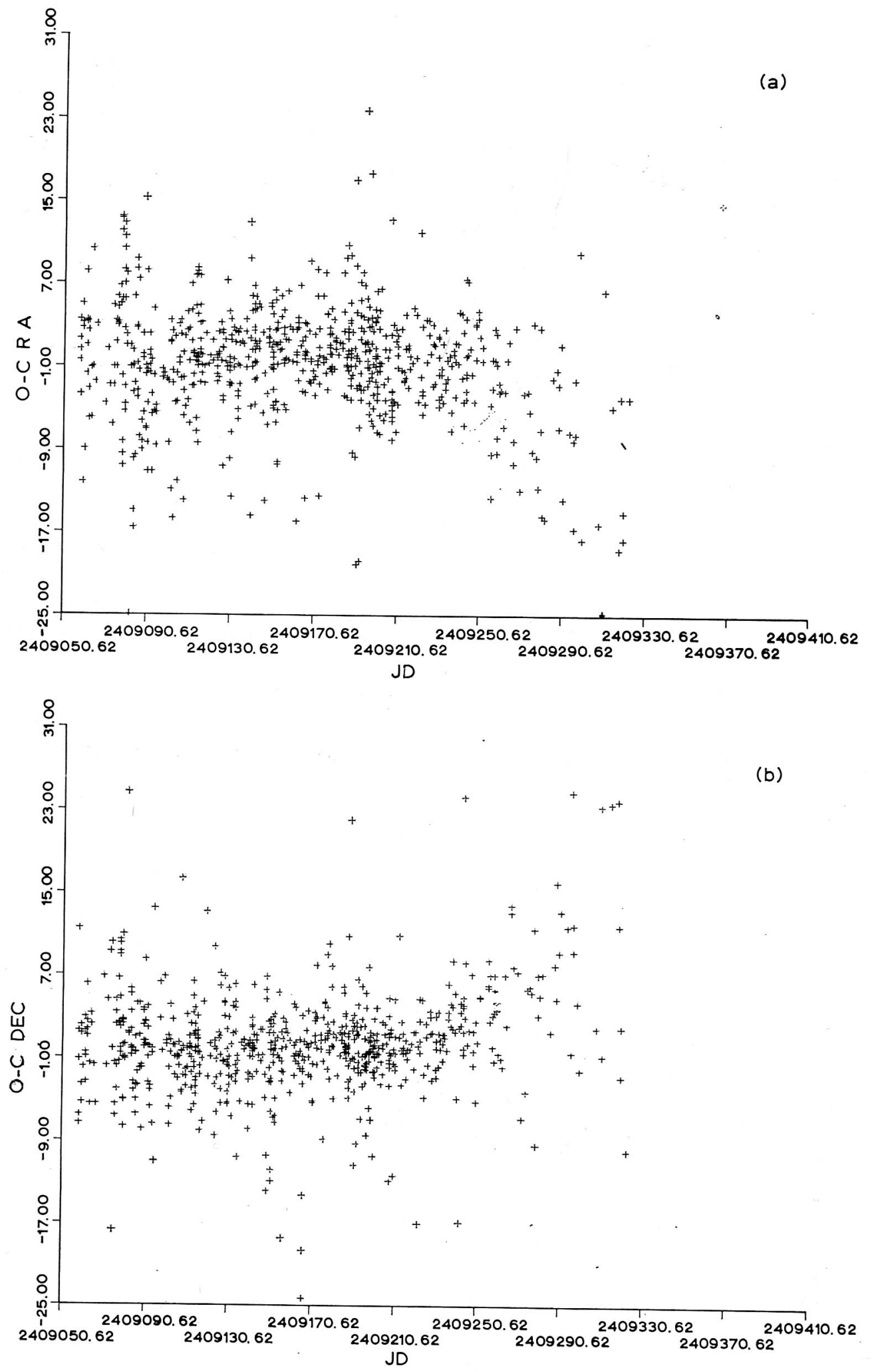

Figs. 2a-b. (O C) residuals in 1883-1884: Case B. 


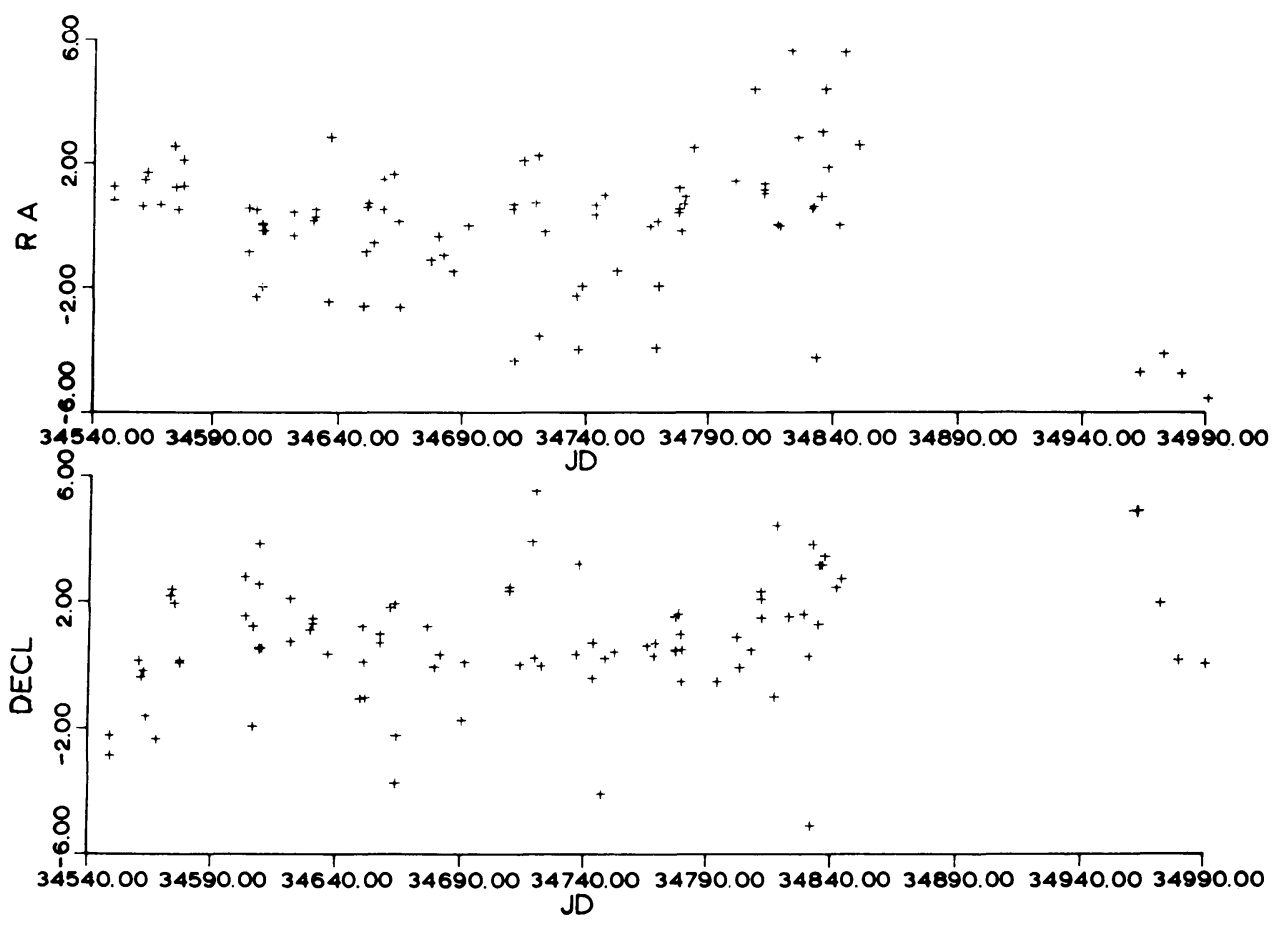

Fig. 3. (O-C) residuals in 1953-1954.

TABLE II

$(\mathrm{O}-\mathrm{C})$ residuals in 1812

\begin{tabular}{lll}
\hline 1812 & $\cos \delta \Delta \alpha$ & $\Delta \delta$ \\
\hline July 23 & -0.05 & $-11^{\prime \prime} 31$ \\
Aug. 6 & -4.25 & -9.02 \\
Aug. 22 & -1.17 & -1.17 \\
Sept. 3 & +3.69 & +2.43 \\
Sept. 14 & +3.22 & +4.10 \\
Sept. 23 & +1.98 & +8.58 \\
\hline
\end{tabular}

\section{Reference}

Herget, P.: 1939, Astron. J. 48, 105. 